\title{
Antitumor effects of cadmium against diethylnitrosamine-induced liver tumors in mice
}

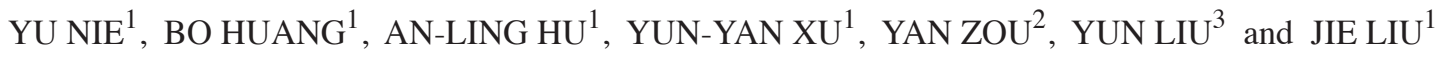 \\ ${ }^{1}$ Key Lab for Basic Pharmacology of Ministry of Education, ${ }^{2}$ School of Public Health, \\ ${ }^{3}$ Guizhou Provincial College-based Key Lab for Tumor Prevention and Treatment with Distinctive Medicines, \\ Zunyi Medical University, Zunyi, Guizhou 563000, P.R. China
}

Received September 7, 2021; Accepted November 2, 2021

DOI: $10.3892 / \mathrm{ol} .2021 .13151$

\begin{abstract}
Cadmium (Cd) has been reported to exhibit antitumor effects against chemically induced liver tumors. However, the antitumor effects of $\mathrm{Cd}$ are not completely understood. Metallotherapy, the use of a toxic metal to attack liver tumors, could be a viable strategy. In the present study, 8-week old, male, C57BL/6 mice were administered injections of diethylnitrosamine (DEN) $(90 \mathrm{mg} / \mathrm{kg}$, and then $50 \mathrm{mg} / \mathrm{kg}$ 2 weeks later), followed by liver tumor promotion with carbon tetrachloride. Cadmium chloride was administered in the drinking water (1000 ppm) from 21-40 weeks after DEN initiation. Body weights were recorded and liver tumor formation was monitored via ultrasound. At the end of experiments, livers were removed, weighed, and the tumor incidence, tumor numbers and tumor size scores were recorded. Liver histology and metallothionein (MT) immunostaining were performed. After DEN injection, animal body weight decreased, and then slowly recovered with time. Cd treatment did not affect animal body weight gain. Ultrasound analysis detected liver tumors 35 weeks after DEN injection, and the mice were necropsied at 40 weeks. Liver/body weight ratios increased in the DEN and DEN + Cd groups. Cd treatment decreased the tumor incidence (71 vs. 17\%), tumor numbers (15 vs. 2) and tumor scores (22 vs. 3) when compared with the DEN only group. Histopathology showed hepatocyte degeneration in all groups, and immunohistochemistry showed MT-deficiency in the liver tumors, while MT staining was intensified in the surrounding
\end{abstract}

Correspondence to: Professor Jie Liu, Key Lab for Basic Pharmacology of Ministry of Education, Zunyi Medical University, 5 Xingpu Road, Zunyi, Guizhou 563000, P.R. China

E-mail: jie@liuonline.com

Professor Yun Liu, Guizhou Provincial College-based Key Lab for Tumor Prevention and Treatment with Distinctive Medicines, Zunyi Medical University, 5 Xingpu Road, Zunyi, Guizhou 563000, P.R. China

E-mail: liuyunzmu@126.com

Key words: cadmium, diethylnitrosamine, carbon tetrachloride, liver tumors, ultrasound, metallothionein tissues. Reverse transcription-quantitative PCR showed increases in $\alpha$-fetoprotein level in DEN-treated livers, and increases in MT-2 and tumor necrosis factor $\alpha(\mathrm{TNF} \alpha)$ levels in Cd-treated livers. Thus, it was concluded that $\mathrm{Cd}$ is effective in the suppression of DEN-induced liver tumors, and that the mechanisms may be related to MT-deficiency in tumors and the induction of TNF $\alpha$ to kill tumor cells.

\section{Introduction}

Hepatocellular carcinoma (HCC) is a common and lethal malignancy (1). Due to a late diagnosis and advanced underlying liver cirrhosis, only limited treatment options with marginal clinical benefits are available for affected patients $(1,2)$. Metallotherapeutics, including platinum and other metal-containing antitumor drugs, is a strategy for treating HCC (3).

Cadmium $(\mathrm{Cd})$ is a toxic heavy metal implicated in carcinogenesis (4) and the development of HCC (5). Similar to the use of chemotherapeutics, such as the carcinogenic and toxic metalloid arsenic, a metallotherapeutic agent could be applied as a toxicant to attack the malignancy. Indeed, $\mathrm{Cd}$ is also effective against HCC in experimental animals (6-8) and in cultured liver tumor SMMC cells and their xenografts $(9,10)$. Cd-coordinated supramolecules, such as Cd-pyrithione (11), Cd telluride/Cd sulfide (12,13), Cd-coordinated thiacalix arene tetrasulfate (14), Cd-thiocarbodiazone complex (15), Cd in combination with human second mitochondria-derived activator of caspase (16) and a novel binuclear hydrazone-based Cd(II) complex (17), have shown potential antitumor effects against chemoresistant malignant cells.

$\mathrm{HCC}$ is often refractory to chemotherapy and radiotherapy at late stages, and is often associated with the loss of metallothionein (MT), probably due to hypermethylation of the MT genes (18-20), and deficiency in MT could make HCC vulnerable to the necrotic effects of $\mathrm{Cd}$, while surrounding normal tissues could be protected through MT induction (6). MTs are small, cysteine-rich cadmium-binding proteins that protect against cadmium toxicity (21).

The present study was initiated in mice to examine the effects of cadmium, administered in drinking water, against HCC formation initiated by diethylnitrosamine (DEN) and promoted by carbon tetrachloride. Small animal ultrasound 
was employed to monitor tumor formation, immunohistochemistry was used to stain for MT, and the expression levels of the HCC biomarker $\alpha$ - fetoprotein, MT and tumor necrosis factor- $\alpha$ in the liver were determined via reverse transcription-quantitative (RT-q)PCR.

\section{Materials and methods}

Reagents. Cadmium chloride $\left(\mathrm{CdCl}_{2}\right), \mathrm{DEN}$ and carbon tetrachloride $\left(\mathrm{CCl}_{4}\right)$ were obtained from MilliporeSigma. Other reagents were of analytical grade.

Animals. Male C57BL/6 mice (6 weeks old) were purchased from the Animal Center Institute of Surgery Research of the Third Military Medical University (Chongqing, China). Animals were maintained in specific pathogen-free facilities at Zunyi Medical University (Zunyi, China), under a controlled environment $\left(22 \pm 1^{\circ} \mathrm{C}, 50 \pm 2 \%\right.$ humidity and a $12: 12 \mathrm{~h}$ light:dark cycle) and free access to purified water and standard laboratory chow. Efforts were made to ameliorate distress and harm to animals by daily monitoring and humane treatment. Animals were adequately cared for, and experimental protocols were in compliance with the Animal Management Guidelines of the Chinese Ministry of Health and approved by the Animal Use and Care Committee of Zunyi Medical University (approval no. 2015-01). The study is reported in accordance with ARRIVE guidelines (https://arriveguidelines.org).

Experimental design. After 2 weeks of acclimation, 40 mice ( 8 weeks of age, weighing 16-19 g) were given the first injection of DEN (90 mg/kg, i.p.). Approximately $80 \%$ of mice survived the first DEN injection, and 2 weeks later, the surviving mice were given the second injection of DEN (50 mg/kg, i.p.), according to the protocol (22). At 4 weeks after the initial DEN challenge, mice (12 weeks of age) were orally administered $20 \% \mathrm{CCl}_{4}, 5 \mathrm{ml} / \mathrm{kg}$, twice a week for 4 months in an attempt to promote liver tumors. At 21 weeks after initial DEN initiation, the mice were randomly divided into the DEN $(n=14)$ and DEN $+\mathrm{Cd}(\mathrm{n}=12)$ group. There was an additional normal control $(\mathrm{n}=5)$ group that did not receive any treatment. Cadmium was administered via the drinking water (1000 ppm) as $\mathrm{CdCl}_{2}$ from 21-40 weeks according to the previous literature $(7,8)$. Body weights were monitored weekly, and a single ultrasound examination of liver tumor formation was performed at 35-39 weeks after DEN initiation. At the end of the 40-week experiment, the mice were anesthetized with sodium pentobarbital $(65 \mathrm{mg} / \mathrm{kg}$, i.p.) and subsequently sacrificed by decapitation. The mouse livers were harvested, and the liver weights and tumor outcomes were recorded. The treatment process is illustrated in Fig. 1A.

The visible tumors were counted and recorded. The tumor incidence (number of tumor-bearing mice), tumor numbers (total tumors found) and tumor size scores (score of 1, tumor size $<1 \mathrm{~mm}$; score of 2, tumor size 1-2 $\mathrm{mm}$; and score of 3 , tumor size $>2 \mathrm{~mm}$ ) were recorded.

Ultrasound detection. Liver tumor formation was measured using B-mode ultrasound $\left(\right.$ Vevo $^{\circledR} 2100$; Fujifilm VisualSonics, Inc.) with $30-\mathrm{MHz}$ peak frequency linear array transducers (MS400; Fujifilm VisualSonics, Inc.; mean beam frequency range of 22-55 MHz) in a digitized scale as previously described (23). Ultrasound was used to detect tumor incidence and size.

Histopathology. Liver tissues were fixed in $10 \%$ buffered formalin for $48 \mathrm{~h}$ at room temperature, embedded in paraffin at $60^{\circ} \mathrm{C}$ and sliced into $3.5-\mu \mathrm{m}$-thick sections using a RM2235 microtome (Leica Microsystems $\mathrm{GmbH}$ ). Sections were deparaffinized in xylene and rehydrated using a gradient of ethanol (100, 95, 85 and 75\%). The histological sections were then stained with hematoxylin and eosin at room temperature for 8-10 min and 4-5 sec, respectively. The digitized images of slices were observed via the Olympus image analysis system (light microscope; Olympus Corporation) at $\mathrm{x} 4$ and x10 magnifications as previously described (24).

Immunohistochemical analysis. Metallothionein (MT) protein retrieval was performed in $10 \mathrm{mmol} / \mathrm{l}$ citrate buffer $\left(\mathrm{pH} 6.0\right.$ ) at $92^{\circ} \mathrm{C}$ for $15 \mathrm{~min}$. Endogenous peroxidase was then blocked with $3 \% \mathrm{H}_{2} \mathrm{O}_{2}$ for $15 \mathrm{~min}$ at room temperature. The tissues were then incubated with $10 \%$ goat non-immune serum (Invitrogen; Thermo Fisher Scientific, Inc.) for $1 \mathrm{~h}$ at room temperature. Subsequently, sections were incubated with MT primary antibodies (cat. no. ab12228, 1:100; Abcam) overnight at $4^{\circ} \mathrm{C}$. After washing with PBS for $5 \mathrm{~min}$, slides were incubated with HRP-conjugated anti-mouse secondary antibody (1:1,000; cat. no. A0216; Beyotime Institute of Biotechnology) for $1 \mathrm{~h}$ at room temperature following the protocol associated with the SABC Detection System (Beyotime). Finally, sections were stained with DAB and counterstained with hematoxylin for $5 \mathrm{~min}$ at room temperature. The digitized images of slices were observed via the LEICA image analysis system (Leica Microsystems $\mathrm{GmbH}$ ) at $\mathrm{x} 10$ magnification. The expression of proteins was quantitatively measured by Image ProPlus 6.0 to obtain the positive staining-integral optical density/area (density mean). A total of four discontinuous areas were used to analyze the expression levels of protein in a blinded fashion as previously described (24).

$R T-q P C R$. Total RNA was extracted from tissues with TRIzol ${ }^{\circledR}$ (Takara Biotechnology Co., Ltd.) and reverse transcribed according to the manufacturer's protocol into cDNA using the PrimeScript ${ }^{\mathrm{TM}}$ RT reagent kit (Takara Biotechnology Co., Ltd.). qPCR was performed utilizing the $\mathrm{iQ}^{\mathrm{TM}}$ SYBR Green Supermix (Bio-Rad Laboratories, Inc.). The PCR cycling conditions were $94^{\circ} \mathrm{C}$ for $3 \mathrm{~min}$, followed by 40 cycles of $94^{\circ} \mathrm{C}$ for $15 \mathrm{sec}, 60^{\circ} \mathrm{C}$ for $20 \mathrm{sec}$ and $72^{\circ} \mathrm{C}$ for $40 \mathrm{sec}$. Data were normalized to $\beta$-actin and expressed using the comparative Cq method $(23,25,26)$. Primers for the mouse genes are shown in Table I.

Statistical analysis. Data are presented as the mean \pm SE and were analyzed by one-way ANOVA, followed by Ducan's multiple comparison test using SigmaPlot (Systat Sofeware, Inc.) version 14. For tumor incidence, Fisher's exact test was performed for the contingency table, while for tumor number and tumor score, data were analyzed with the Kruskal-Wallis test, followed by Dunn's post hoc test. $\mathrm{P}<0.05$ was considered to indicate a statistically significant difference. 
Table I. Primer sequences for reverse transcription-quantitative PCR.

\begin{tabular}{llll}
\hline Name & Access ID & Forward primer (5'-3') & Reverse primer (5'-3') \\
\hline AFP & NM_007423 & AGCAGGACTGCTCGAAACAT & AGCGAAATGTAGCAGGAGGA \\
$\beta$-actin & NM_007393 & GATCTGGCACCACACCTTCT & GGGGTGTTGAAGGTCTCAAA \\
MT-2 & NM_008630 & CCGATCTCTCGTCGATCTTC & AGGAGCAGCAGCTTTTCTTG \\
TNF- $\alpha$ & NM_013693 & TAGCCAGGAGGGAGAACAGA & TTTTCTGGAGGGAGATGTGG
\end{tabular}

AFP, $\alpha$-fetoprotein; MT, mettalothionein; TNF $\alpha$, tumor necrosis factor $\alpha$.
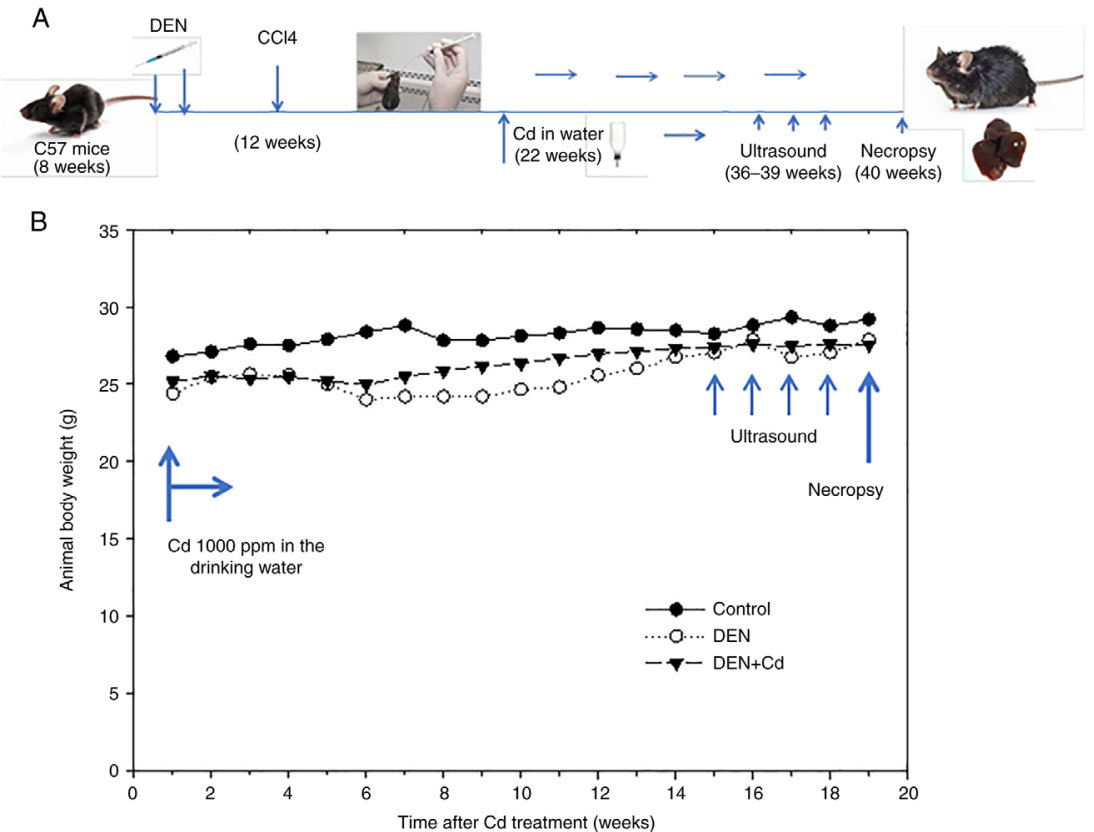

Figure 1. Animal body weights and Cd treatment procedures. (A) Experimental design. (B) Time after Cd treatment. At 21 weeks after DEN initiation, mice were administered Cd (500 ppm in the drinking water) for 19 weeks (week 40 of DEN initiation). Data represent the mean of the control ( $\mathrm{n}=5)$, DEN ( $\mathrm{n}=14)$ and DEN + Cd ( $\mathrm{n}=12)$ groups. DEN, dimethylnitrosamine; $\mathrm{Cd}$, cadmium; $\mathrm{CCl}_{4}$, carbon tetrachloride.

\section{Results}

Animal survival and body weight. After the initial DEN (90 mg/kg, i.p.) administration, $80 \%$ of mice survived within 7 days, with a $20 \%$ reduction in body weight. After the second DEN injection, $95 \%$ of mice survived, with a $\sim 15 \%$ reduction in body weight. Animal body weights slowly recovered afterwards over time. $\mathrm{CCl}_{4}$ promotion $(20 \%$, $5 \mathrm{ml} / \mathrm{kg}$, p.o., twice/week) was started at week 4 post-DEN injection and continued for 4 months. Cd was administered from 21-40 weeks after DEN initiation through the drinking water $(1000 \mathrm{ppm})$ for 19 weeks. All mice survived Cd treatment. Ultrasound was randomly performed on a few mice at week 36 post-DEN injection (15 weeks after Cd treatment), 4 times, until week 40 post-DEN initiation (19 weeks after $\mathrm{Cd}$ treatment). The animal body weights after $\mathrm{Cd}$ intervention are shown in Fig. 1B.

Ultrasound detection of liver tumor formation. Small animal ultrasound analysis was used to monitor tumor formation 30 weeks after DEN injection, but no tumors were detected. At 36 weeks after DEN injection, liver tumors with small sizes were detected at an $\sim 40 \%$ incidence rate. At week 40 after DEN initiation, a $65 \%$ tumor incidence rate was recorded. Fig. 2 shows representative ultrasound images of DEN-induced liver tumors at 40 weeks after DEN initiation in control, DEN- and $\mathrm{DEN}+\mathrm{Cd}$-treated mice. In the control mice, the ultrasound showed normal liver, while in the DEN-treated mice, the liver density was increased (arrows), indicative of liver tumors. In the DEN + Cd-treated mice, only one mouse showed a detectable tumor with decreased density compared with non-tumor tissues.

Animal liver/body weight ratio. At the end of experiment, the mice were euthanized and their livers were collected and weighed, and the liver/body ratios were calculated. Fig. 3 shows the liver/body weight ratios of the three groups. DEN increased the liver/body weight ratio from 48.5 to $53.4 \mathrm{mg} / \mathrm{g}$, while DEN + Cd further increased the liver/body weight ratio to $55.9 \mathrm{mg} / \mathrm{g}$, which were significantly higher results compared with the controls.

Liver tumor outcome. Representative gross liver images are shown in Fig. 4, where liver tumors were detected in 


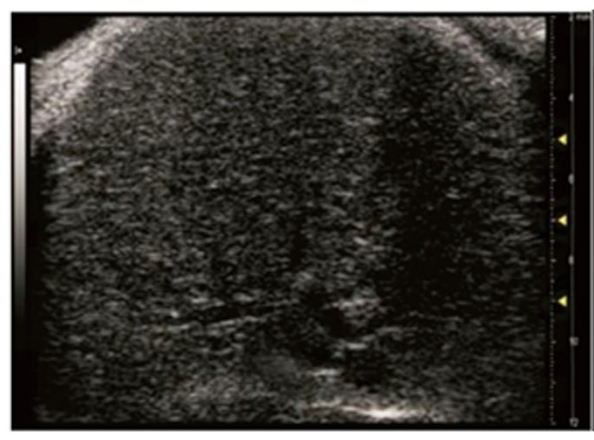

Control

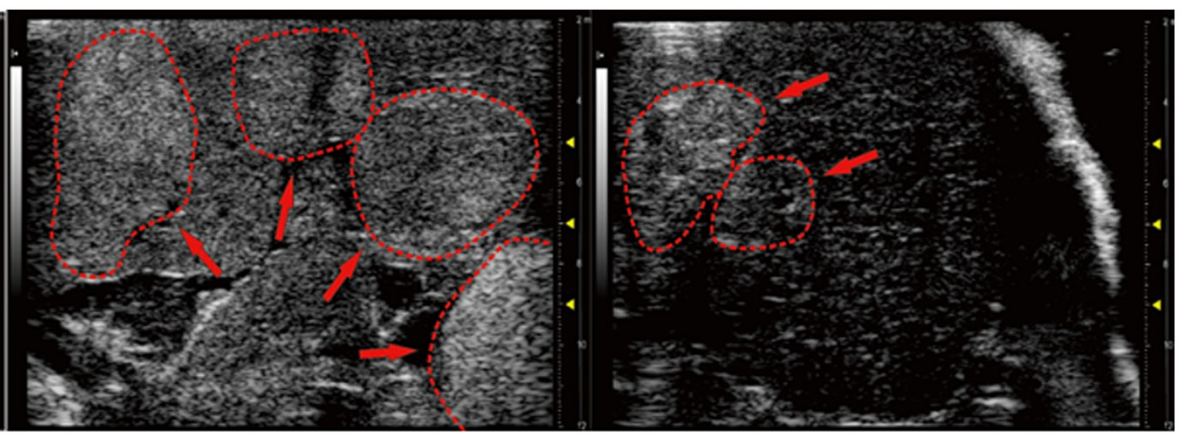

DEN
$\mathrm{DEN}+\mathrm{Cd}$

Figure 2. Representative ultrasound images of liver tumors. The mice were anesthetized, and after fur removal, the liver tumor imaging was performed by ultrasound. Arrows indicate the ultrasound detection area indicating the formation of liver tumors. DEN, dimethylnitrosamine; Cd, cadmium.

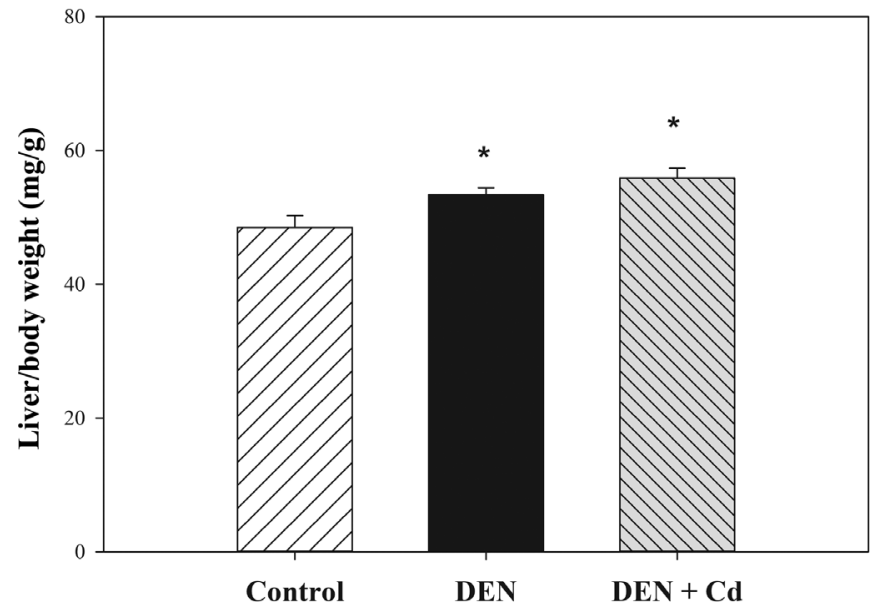

Figure 3. Animal liver/body weight ratios at necropsy. At 16 weeks after DEN injection, the mice were euthanized, and their livers were collected and weighed. The liver/body weight ratios of the control $(n=5), D E N(n=4)$ and Cd $(n=12)$ are shown. Data are presented as the mean $\pm \mathrm{SE}$. ${ }^{*} \mathrm{P}<0.05$ vs. control. DEN, dimethylnitrosamine; Cd, cadmium.

DEN-treated mice, no tumors were found in control mice and only a few tumors were found in DEN + Cd-treated mice.

The tumor outcomes, recorded from blinded observations, are listed in Table II. In total, 10 out of 14 DEN-treated mice had tumors, with a tumor incidence of $71 \%$, and only 2 out of $12 \mathrm{DEN}+\mathrm{Cd}$-treated mice had tumors, with a tumor incidence of $17 \%$; a total of 15 tumors were found in DEN-treated mice, but only 2 tumors in DEN + Cd-treated mice. Some tumors in the DEN-treated mice were large, and the tumor score was 22, while in DEN + Cd-treated mice, the score was 3. The tumor number and tumor score of the DEN $+\mathrm{Cd}$ group were statistically significant compared with the DEN group.

Histology and immunohistochemistry. Fig. 5 shows representative histology and immunochemistry images of the tissues in the different groups. Liver tumors were observed in DEN-treated mice, and hepatocellular degeneration was evident in both DEN- and DEN + Cd-treated mice. In DEN-treated livers, MT staining was not present in the liver tumors, but was strongly present in the tissues surrounding the tumors. DEN + Cd-treated mice exhibited increased intensity of the MT stain.
Table II. Tumor outcomes of DEN and DEN + Cd groups.

\begin{tabular}{lrccc}
\hline Group & $\mathrm{n}$ & $\begin{array}{c}\text { Tumor } \\
\text { incidence, } \mathrm{n}(\%)\end{array}$ & $\begin{array}{c}\text { Tumor } \\
\text { number }\end{array}$ & $\begin{array}{c}\text { Tumor } \\
\text { score }\end{array}$ \\
\hline Control & 5 & 0 & 0 & 0 \\
DEN & 14 & $10(71)$ & 15 & 22 \\
DEN $+\mathrm{Cd}$ & 12 & $2^{\mathrm{a}}(17)$ & $2^{\mathrm{a}}$ & $3^{\mathrm{a}}$ \\
\hline
\end{tabular}

${ }^{\mathrm{a}} \mathrm{P}<0.05$ vs. DEN. DEN, dimethylnitrosamine; $\mathrm{Cd}$, cadmium.

RT-qPCR analysis of liver gene expression. Fig. 6 shows the expression of liver genes in the control mice $(n=5)$, and in the mice treated with DEN $(n=14)$ and $\mathrm{DEN}+\mathrm{Cd}(\mathrm{n}=12)$, with controls set at $100 \%$. DEN treatment increased the expression of $\alpha$-fetoprotein (AFP) over 2 -fold (242\% of control); however, due to huge individual variance, the difference was not statistically significant. DEN + Cd treatment increased the expression to $112 \%$ of the control. The expression of MT-2 was slightly decreased by DEN treatment (73\% of the control), but significantly increased by DEN $+\mathrm{Cd}$ treatment by almost 3 -fold (294\% of the control). The expression of tumor necrosis factor $\alpha(\mathrm{TNF} \alpha)$ was slightly increased by DEN treatment ( $142 \%$ of the control), but significantly increased by DEN + Cd treatment ( $224 \%$ of the control).

\section{Discussion}

The present study demonstrated the antitumor effects of cadmium against DEN-initiated and $\mathrm{CCl}_{4}$-promoted $\mathrm{HCC}$, as evidenced by ultrasound imaging, tumor incidence, tumor number and tumor score. Immunohistochemistry showed MT was deficient in HCC, but was induced in the tissues surrounding the tumor. RT-qPCR revealed that the HCC biomarker AFP was increased in $\mathrm{HCC}$, but attenuated by $\mathrm{Cd}$, although the changes were not statistically significant. $\mathrm{Cd}$ treatment also induced MT and TNF $\alpha$ in the liver. However, histology showed hepatocyte degeneration in the liver in both DEN- and DEN + Cd-treated groups.

Cadmium has previously been shown to exert antitumor effects against DEN-induced HCC in B6C3F1 mice (6-8), 


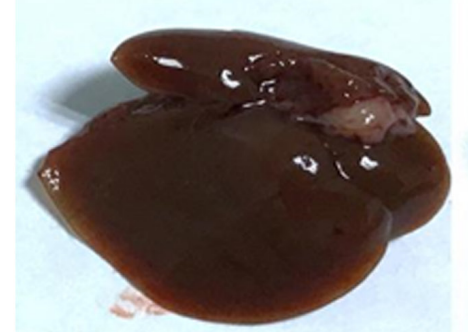

Control

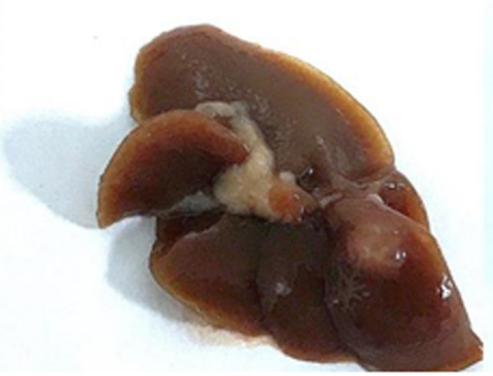

DEN

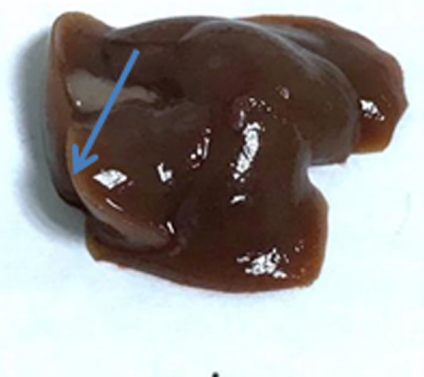

$\mathrm{DEN}+\mathrm{Cd}$

Figure 4. Representative gross liver images at necropsy. Tumors were more visible in DEN only group compared with the DEN + Cd group. The arrow indicates the liver tumor in the DEN group. DEN, dimethylnitrosamine; Cd, cadmium.

\section{Control}
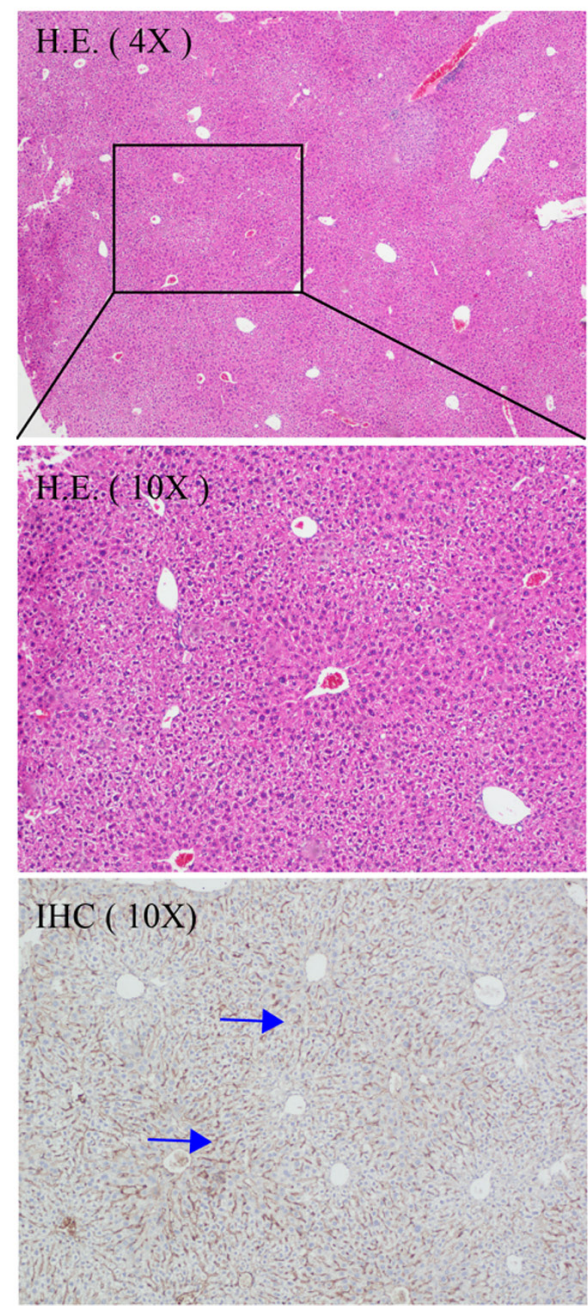

DEN
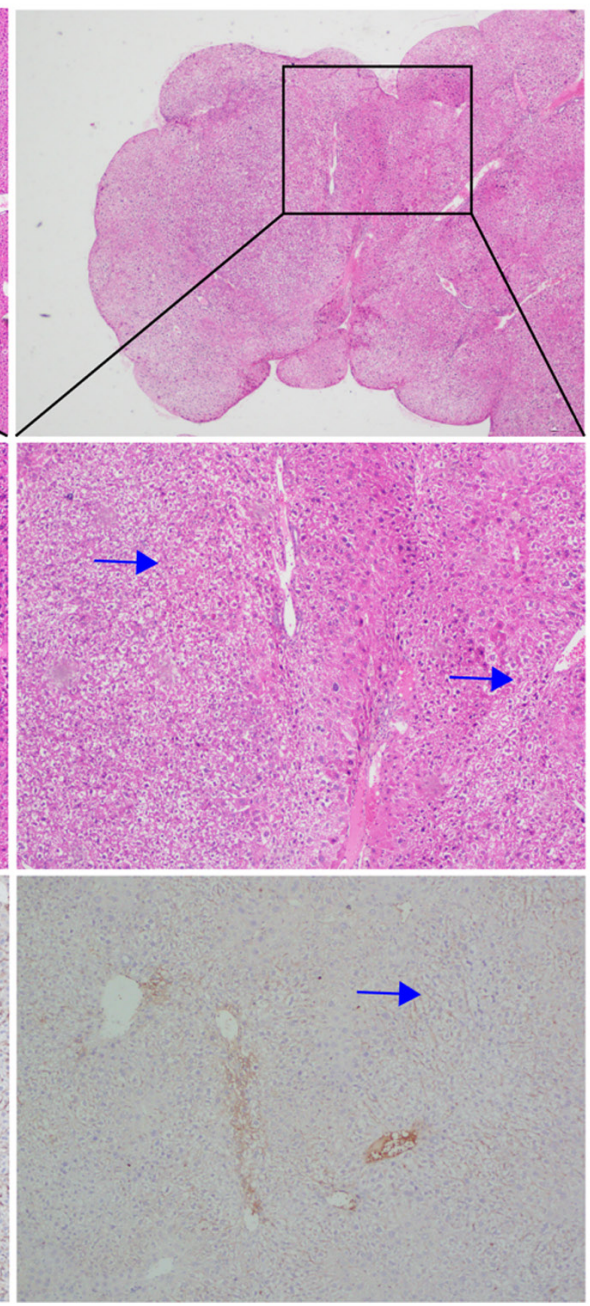

$\mathrm{DEN}+\mathrm{Cd}$

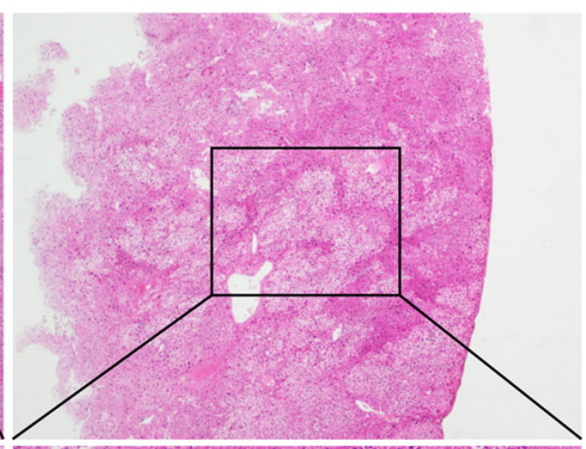

Figure 5. Representative HE and MT immunohistochemistry images. The top panel shows low power (x4 magnification) HE images and the middle panels show enlarged (x10 magnification) versions of the inset box. The bottom panels show MT immunostaining (x10 magnification) images. Arrows in the HE images indicate liver tumors, while arrows in the immunostaining images indicate MT staining. HE, hematoxylin and eosin; IHC, immunohistochemistry; MT, metallothionein; DEN, dimethylnitrosamine; Cd, cadmium.

including the late stage of HCC by inducing tumor necrosis (6). The present study replicated these previous findings in C57/BL mice. Cd exposure is known to produce tumors in the lungs, liver, prostate, pancreas and injection sites (4), and long-term Cd exposure is implicated in HCC development (5). Paradoxically, in an effort to promote DEN-initiated HCC, Cd was unexpectedly found to produce antitumor effects (7). This phenomenon is common for metallo-chemotherapy agents. For 


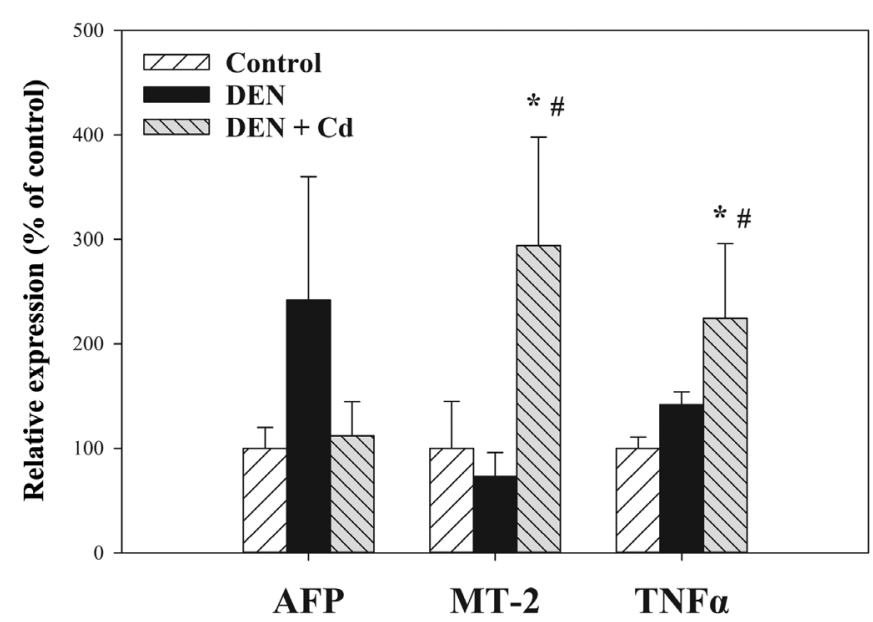

Figure 6. Expression of AFP, MT-2, and TNF $\alpha$ via RT-qPCR. At 19 weeks after $\mathrm{Cd}$ treatment (500 ppm in the drinking water), hepatic total RNA was extracted and subjected to RT-qPCR analysis. Data are presented as the mean \pm SEM of the control $(n=5)$, DEN $(n=14)$ and DEN $+C d(n=12)$ groups. ${ }^{*} \mathrm{P}<0.05$ vs. control; ${ }^{*} \mathrm{P}<0.05$ vs. DEN. AFP, $\alpha$-fetoprotein; MT, mettalothionein; RT-qPCR, reverse transcription-quantitative PCR; DEN, dimethylnitrosamine; $\mathrm{Cd}$, cadmium; TNF $\alpha$, tumor necrosis factor $\alpha$.

example, arsenic is a well-known human carcinogen, but it is also effective against hematological malignancies and certain solid tumors with arsenic-metal complexes (27), and the toxic effects of Cd towards colorectal carcinoma cells (28) might contribute to the antitumor effects of $\mathrm{Cd}$. The use of a toxic metal/metalloid to treat chemo-resistant malignancies could be a strategy in metallo-chemotherapy.

Non-invasive imaging of HCC growth in mice with ultrasound technology (29) would help monitor HCC growth, the treatment efficacy and the treatment duration. We have previously successfully applied ultrasound in cardiovascular studies (23). In the present study, HCC formation could be detected in mice at 4 months after DEN initiation (at 6 months of age), while at 8 months of age, $50 \%$ tumor incidence was detected, and at 9 months of age, $65 \%$ tumor incidence was detected, which was consistent with the necropsy findings at the end of experiment (at 10 months of age). Tumor promotion with $\mathrm{CCl}_{4}$ in the current study resulted in higher tumor incidence compared with that found in previous studies using $\mathrm{Cd}$ alone $(7,8)$. With the aid of ultrasound monitoring, HCC-bearing mice were sacrificed at 40 weeks of age to examine $\mathrm{Cd}$ antitumor effects, with findings of decreased incidence (71 vs. 17\%), decreased tumor numbers (15 vs. 2) and decreased tumor size scores (22 vs. 3) compared with the DEN-treated group. Ultrasound appears to be a sensitive means to monitor liver tumor development and helped to define the $\mathrm{Cd}$ treatment regimen for tumor growth inhibition in the present study.

MTs are small, cysteine-rich cadmium-binding proteins that protect against cadmium toxicity and are encoded by MT isoform genes $(21,30)$. In studies on human HCC, MT-1G was hypermethylated, leading to decreased expression (19), MT-1X and MT-2A tended to decrease with the progression of HCC (20), and at the late stage of HCC, MT-1A, MT-2A and metal regulatory transcription factor 1 levels were decreased (25). In the present study, immunostaining for MT was not detected in HCC, while the surrounding tissues exhibited intense staining for MT. This molecular event could be turned into an advantage for Cd to selectively kill HCC, while saving normal cells that will be protected from $\mathrm{Cd}$ cytotoxicity by the MT induction.

AFP is a well-known biomarker of HCC that exhibits increased levels in DEN-treated mouse livers (31). In the present study, the expression of AFP in the Cd-treated group was attenuated, consistent with the antitumor effects of $\mathrm{Cd}$. By contrast, the expression of MT-2 and TNF $\alpha$ in the Cd-treated group was higher compared with that in the DEN model group. Cd is a potent inducer of the MT genes; both MT-1 and MT-2 are coordinately expressed in the mouse liver (21), and increased MT-2 expression could protect against $\mathrm{Cd}$ toxicity. Induction of $\mathrm{TNF} \alpha$ by $\mathrm{Cd}$ plays a dual role in killing tumor cells, as well as in producing liver damage (32).

It should be noted that the antitumor effects of $\mathrm{Cd}$ should be carefully assessed to ensure the balance between the benefits and risks from its use. Necrotic effects of $\mathrm{Cd}$ on $\mathrm{HCC}$ are a desired therapeutic outcome, but $\mathrm{Cd}$-induced liver injury is an undesired toxic effect. In the present study, the antitumor effects of $\mathrm{Cd}$ were accompanied by liver damage to various degrees (Figs. 4 and 5). Caution should be taken when using $\mathrm{Cd}$ to treat malignancies over a long period of time, and close monitoring of the potential adverse effects to balance efficacy and toxicity is important.

Collectively, the present study demonstrated the antitumor effects of Cd against DEN-induced HCC in C57/B6 mice; the mechanism of action appeared to be related to MT-deficiency in HCC, while normal surrounding tissues were protected by MT. Thus, the targeting of MT-deficiency in HCC could be a potential therapeutic strategy.

\section{Acknowledgements}

Not applicable.

\section{Funding}

This study was supported by the Guizhou Provincial Science and Technology Program [grant no. QKH(2019)1346], the Science and Technology Talent Support Project of the Educational Department of Guizhou Province [grant no. KY(2018)055], the Innovation Talent Team of Zunyi [grant no.ZSKRC(2019)1], the Innovation Talent Team of Guizhou Science and Technology Department [grant no. QKHPTRC(2020)5007] and the Science and Technology Plan of Zunyi [grant no. ZSKRCPT(2020)7].

\section{Availability of data and materials}

The datasets used and/or analyzed during the current study are available from the corresponding author on reasonable request.

\section{Authors' contributions}

YL, JL and YN conceived the experiment. YN performed the animal studies, including drug administration, body weight recording and necropsy, and qPCR and histology analyses. $\mathrm{BH}$ and YYX performed the small animal ultrasound imaging, ALH performed the pathology and immunohistochemistry analyses, and YZ performed the statistical analysis. YL, YN, 
BH, YYX and ALH confirm the authenticity of all the raw data. All authors have read and approved the final manuscript.

\section{Ethics approval and consent to participate}

All animal care and experimental protocols complied with the Animal Management Guidelines of the Chinese Ministry of Health and were approved by the Animal Use and Care Committee of Zunyi Medical University (Zunyi, China; approval no. 2015-01).

\section{Patient consent for publication}

Not applicable.

\section{Competing interests}

The authors declare that they have no competing interests.

\section{References}

1. Wörns MA and Galle PR: Future perspectives in hepatocellular carcinoma. Dig Liver Dis 42 (Suppl 3): S302-S309, 2010.

2. Javan H, Dayyani F and Abi-Jaoudeh N: Therapy in Advanced Hepatocellular Carcinoma. Semin Intervent Radiol 37: 466-474, 2020.

3. Kaluderović GN and Paschke R: Anticancer metallotherapeutics in preclinical development. Curr Med Chem 18: 4738-4752, 2011.

4. Waalkes MP: Cadmium carcinogenesis. Mutat Res 533: 107-120, 2003.

5. Satarug S: Long-term exposure to cadmium in food and cigarette smoke, liver effects and hepatocellular carcinoma. Curr Drug Metab 13: 257-271, 2012.

6. Waalkes MP, Diwan BA, Rehm S, Ward JM, Moussa M, Cherian MG and Goyer RA: Down-regulation of metallothionein expression in human and murine hepatocellular tumors: Association with the tumor-necrotizing and antineoplastic effects of cadmium in mice. J Pharmacol Exp Ther 277: 1026-1033, 1996.

7. Waalkes MP, Diwan BA, Weghorst CM, Bare RM, Ward JM and Rice JM: Anticarcinogenic effects of cadmium in B6C3F1 mouse liver and lung. Toxicol Appl Pharmacol 110: 327-335, 1991.

8. Waalkes MP, Diwan BA, Weghorst CM, Ward JM, Rice JM, Cherian MG and Goyer RA: Further evidence of the tumor-suppressive effects of cadmium in the B6C3F1 mouse liver and lung: Late stage vulnerability of tumors to cadmium and the role of metallothionein. J Pharmacol Exp Ther 266: $1656-1663,1993$.

9. Du H, Liu X, Liu Y, Jin M, Huang W and Sun Z: Inhibition effect of cadmium chloride on human hepatocellular carcinoma cells SMMC7721. Chin J Publ Health 22: 194-195, 2006.

10. Du HJM, Liu Y, Liu X, Wang W and Sun Z: Study on the anti-tumor effect of calcium chloride in vivo. Mod Prev Med 35: 3763-3765, 2008.

11. Chen X, Wu J, Yang Q, Zhang X, Zhang P, Liao S, He Z, Wang X, Zhao C and Liu J: Cadmium pyrithione suppresses tumor growth in vitro and in vivo through inhibition of proteasomal deubiquitinase. Biometals 31: 29-43, 2018.

12. Xu N, Piao M, Arkin K, Ren L, Zhang J, Hao J, Zheng Y and Shang Q: Imaging of water soluble $\mathrm{CdTe} / \mathrm{CdS}$ core-shell quantum dots in inhibiting multidrug resistance of cancer cells. Talanta 201: 309-316, 2019

13. Zhang G, Shi L, Selke M and Wang X: CdTe quantum dots with daunorubicin induce apoptosis of multidrug-resistant human hepatoma HepG2/ADM cells: In vitro and in vivo evaluation. Nanoscale Res Lett 6: 418, 2011

14. Zhou X, Koizumi Y, Zhang M, Natsui M, Koyota S, Yamada M, Kondo Y, Hamada F and Sugiyama T: Cadmium-coordinated supramolecule suppresses tumor growth of T-cell leukemia in mice. Cancer Sci 106: 635-641, 2015.
15. Pérez JM, Cerrillo V, Matesanz AI, Millán JM, Navarro P, Alonso $\mathrm{C}$ and Souza P: DNA interstrand cross-linking efficiency and cytotoxic activity of novel cadmium(II)-thiocarbodiazone complexes. ChemBioChem 2: 119-123, 2001.

16. Guo C, Li Y, Zhang H, Wang Z, Jin M, Zhang L, An L, Hu G, Liu X, Liu Y, et al: Enhancement of antiproliferative and proapoptotic effects of cadmium chloride combined with hSmac in hepatocellular carcinoma cells. Chemotherapy 57: 27-34, 2011.

17. Bjelogrlić S, Todorović TR, Cvijetić I, Rodić MV, Vujčić M, Marković S, Araškov J, Janović B, Emhemmed F, Muller CD, et al: A novel binuclear hydrazone-based Cd(II) complex is a strong pro-apoptotic inducer with significant activity against $2 \mathrm{D}$ and 3D pancreatic cancer stem cells. J Inorg Biochem 190: 45-66, 2019.

18. Jacob ST, Majumder S and Ghoshal K: Suppression of metallothionein-I/II expression and its probable molecular mechanisms. Environ Health Perspect 110 (Suppl 5): 827-830, 2002.

19. Kanda M, Nomoto S, Okamura Y, Nishikawa Y, Sugimoto H, Kanazumi N, Takeda S and Nakao A: Detection of metallothionein $1 \mathrm{G}$ as a methylated tumor suppressor gene in human hepatocellular carcinoma using a novel method of double combination array analysis. Int J Oncol 35: 477-483, 2009.

20. Tao X, Zheng JM, Xu AM, Chen XF and Zhang SH: Downregulated expression of metallothionein and its clinicopathological significance in hepatocellular carcinoma. Hepatol Res 37: 820-827, 2007.

21. Klaassen CD, Liu J and Choudhuri S: Metallothionein: An intracellular protein to protect against cadmium toxicity. Annu Rev Pharmacol Toxicol 39: 267-294, 1999.

22. Yi X, Long L, Yang C, Lu Y and Cheng M: Maotai ameliorates diethylnitrosamine-initiated hepatocellular carcinoma formation in mice. PLoS One 9: e93599, 2014

23. Huang B, Hu P, Hu A, Li Y, Shi W, Huang J, Jiang Q, Xu S, Li L and $\mathrm{Wu}$ Q: Naringenin attenuates carotid restenosis in rats after balloon injury through its anti-inflammation and anti-oxidative effects via the RIP1-RIP3-MLKL signaling pathway. Eur J Pharmacol 855: 167-174, 2019.

24. Hu A, Huang J, Li S, Gao Y, Wu L, Deng J, Liu J, Gong Q, $\mathrm{Li} \mathrm{L}$ and $\mathrm{Xu} \mathrm{S}$ : Involvement of stromal cell-derived factor-1a (SDF-1 $\alpha$ ), stem cell factor (SCF), fractalkine (FKN) and VEGF in TSG protection against intimal hyperplasia in rat balloon injury. Biomed Pharmacother 110: 887-894, 2019.

25. Li H, Lu YF, Chen H and Liu J: Dysregulation of metallothionein and circadian genes in human hepatocellular carcinoma. Chronobiol Int 34: 192-202, 2017.

26. Livak KJ and Schmittgen TD: Analysis of relative gene expression data using real-time quantitative PCR and the 2(-Delta Delta C(T)) Method. Methods 25: 402-408, 2001.

27. Fei W, Li C, Tao J, Cai X, Yao W, Ye Y, Zhang Y, Yao Y, Song Q, Li F, et al: Construction of arsenic-metal complexes loaded nanodrugs for solid tumor therapy: A mini review. Int J Pharm 583: 119385, 2020.

28. Iftode A, Drăghici GA, Macașoi I, Marcovici I, Coricovac DE, Dragoi R, Tischer A, Kovatsi L, Tsatsakis AM, Cretu O, et al: Exposure to cadmium and copper triggers cytotoxic effects and epigenetic changes in human colorectal carcinoma HT-29 cells. Exp Ther Med 21: 100, 2021.

29. Anton N,Parlog A,Bou About G, Attia MF, Wattenhofer-Donzé M, Jacobs H, Goncalves I, Robinet E, Sorg T and Vandamme TF: Non-invasive quantitative imaging of hepatocellular carcinoma growth in mice by micro-CT using liver-targeted iodinated nano-emulsions. Sci Rep 7: 13935, 2017.

30. Klaassen CD, Liu J and Diwan BA: Metallothionein protection of cadmium toxicity. Toxicol Appl Pharmacol 238: 215-220, 2009.

31. Ghufran H, Azam M, Mehmood A, Butt H and Riazuddin S: Standardization of diethylnitrosamine-induced hepatocellular carcinoma rat model with time based molecular assessment. Exp Mol Pathol 123: 104715, 2021.

32. Kayama F, Yoshida T, Elwell MR and Luster MI: Role of tumor necrosis factor-alpha in cadmium-induced hepatotoxicity. Toxicol Appl Pharmacol 131: 224-234, 1995.

This work is licensed under a Creative Commons Attribution-NonCommercial-NoDerivatives 4.0 International (CC BY-NC-ND 4.0) License. 